\title{
Discrete Wavelet Transform Based Image Fusion Using Unsharp Masking
}

\author{
Sumanth Kumar Panguluri ${ }^{1 *}$, Laavanya Mohan \\ 1 Department of Electronics and Communication Engineering, Vignan's Foundation for Science, Technology, and Research, \\ Vadlamudi, Guntur, Andhra Pradesh, 522213, India \\ * Corresponding author, e-mail: skp6472@gmail.com
}

Received: 17 July 2019, Accepted: 14 October 2019, Published online: 02 December 2019

\begin{abstract}
Nowadays the result of infrared and visible image fusion has been utilized in significant applications like military, surveillance, remote sensing and medical imaging applications. Discrete wavelet transform based image fusion using unsharp masking is presented. DWT is used for decomposing input images (infrared, visible). Approximation and detailed coefficients are generated. For improving contrast unsharp masking has been applied on approximation coefficients. Then for merging approximation coefficients produced after unsharp masking average fusion rule is used. The rule that is used for merging detailed coefficients is max fusion rule. Finally, IDWT is used for generating a fused image. The result produced using the proposed fusion method is providing good contrast and also giving better performance results in reference to mean, entropy and standard deviation when compared with existing techniques.
\end{abstract}

\section{Keywords}

infrared image, visible image, image fusion, DWT, unsharp masking

\section{Introduction}

Nowadays sensor technology is rapidly growing. The same scene information is captured using infrared and visible image cameras. Infrared cameras capture the thermal radiation of light. Infrared image main advantage is, it provides good target details even in poor weather conditions such as low light condition, snow, and fog. But the problem is, it is having low spatial resolution and poor texture [1]. Background information is not clearly seen in infrared images. On the other side, the visible camera captures the reflection of light. It is having high spatial resolution [2]. The visible image's main advantage is, it provides good background information so that humans can easily understand scene information. But the problem is, it cannot capture target details in poor weather conditions. By considering infrared and visible images advantages. Fusing them provides better scene information in a single image [3]. The result of fusion has been utilized in most significant applications such as the military [4], surveillance [5], remote sensing [6], and medical imaging applications [7].

Traditionally fusion can be performed mainly using two methods.

1. Spatial domain fusion method.

2. Transform domain fusion method.
In the spatial domain method, two source images (infrared, visible) are combined based on fusion rule. Here no transforms are used in this method. Fusion rule is applied directly to pixels of the images. Generally, fusion rules used in this method are the average and max fusion rules. The fused image result that has been produced based on the spatial domain based method has low contrast, reduction in sharpness and blurred edges [8]. Later transform domain fusion based method has been developed for improving the performance of the fusion result. The problems that occur in the spatial domain fusion method have been rectified by using the transform domain fusion method [9].

Mainly transform domain fusion based method involves three steps.

1. In the first step, a multi-scale transform has been used for decomposing two source images (infrared, visible) into multi-scale components. (Highfrequency and low-frequency components).

2. Different fusion rules have been used for combining multi-scale components.

3. The final fused image has been reconstructed with an inverse multi-scale transform. 
Different multi-scale transforms such as DWT [10], SWT [11], and DCT [12] have been used for fusing infrared and visible images. Although transform domain based fusion methods have advantages compared to the spatial domain fused based method but still have limitations such as they cannot preserve edge and curve information [13].

\section{Related works}

Li et al. [14] have introduced a multi-sensor fusion technique based on DWT. For merging approximation and detailed coefficients of input images the max fusion rule has been used. Feature selection has been done by using maximum area selection based rule and through consistency verifying step. The proposed technique fused image has been giving better performance compared to laplacian pyramid techniques because of directional selectivity, compactness, and orthogonality of DWT.

Fan et al. [15] have presented an improved fusion method based on DWT. For merging approximation coefficients, the human visual system (HVS) based fusion rule is used. For merging detailed coefficients absolute value fusion rule has been used. IDWT has been used for reconstructing the fused image. The resultant image obtained using this method is preserving edge details of the target better than the mean method, min method, and mean max method.

Godse and Bormane [16] have presented a fusion technique based on DWT. The fusion rule that has been used in this technique is the maximum selection rule. The fused image is providing better performance but producing a blurred result.

$\mathrm{Xu}$ and $\mathrm{Su}$ [17] have introduced the fusion algorithm based on discrete wavelet decomposition. The low-frequency coefficient of the infrared image is improved in terms of contrast and gradient by using the enhanced method using DWT and DT-CWT. Fused image produced using this method has improved contrast when compared with basic DWT and DTCWT methods.

Zhou and Tan [18] have introduced the fusion technique for multimodal images (infrared, visible) that is based on the wavelet transform. Fusion strategies that have been used are developed using a comparison of different fusion rules. The Fused image is providing a better overall understanding of the scene information but unable to produce good texture information.

Vijayarajan and Muttan [19] have presented a fusion scheme based on DWT. Principle component analysis has been utilized for merging approximation and detail coefficients. PCA fusion is based on the average of principal components of coefficients of source images. This fusion scheme is clearly removing spatial distortions.

Han et al. [20] have presented a fusion scheme for complementary images (infrared, visible) based on DWT. The wavelet decomposition technique is applied to input images to produce approximation and detail coefficients. Approximation coefficients have been merged using absolute value selection fusion rule and detailed coefficients have been merged using weighted average fusion rule. IDWT has been used for reconstructing the fused image. Compared to traditional methods fused image achieved with the above scheme has given good performance regarding entropy, spatial frequency, average gradient, and standard deviation but it lacks in achieving good contrast.

Zhan et al. [10] have presented a fusion method for multi-sensor images (infrared, visible) based on DWT. Regional energy fusion strategy has been used for merging approximation coefficients. The fused image achieved using the above method has given better target information but lacks in providing good texture information.

Habeeb et al. [21] have presented an enhanced fusion method based on weighted averaging or a discrete wavelet transform. Input images (infrared, visible) that have fused in the spatial domain using a weighted average fusion rule produce a blurred image. And input images that have fused in transform domain basing on DWT produce a better result but it reduces spatial domain details. Applying a sharpening filter on input images in the spatial domain and fusing using weighted averaging or discrete wavelet transform improves the contrast details of the resultant fused image.

Habeeb et al. [22] have presented a multi-modal fusion algorithm. The main techniques used for enhancement are PCA, Histogram equalization and sharpen filter. The fusion rule used is weighted average rule. Proposed algorithm got better results than the existing methods.

The method proposed here is a discrete wavelet transform based image fusion using unsharp masking. This method is aimed to give better performance compared to multi-scale transform techniques. Unsharp masking has been used for improving contrast. The proposed method has been developed for improving contrast and also preserving edge information.

\section{Proposed method}

Discrete wavelet transform based image fusion using unsharp masking is presented. Mainly in this method, the first two source images (infrared, visible) are scaled down to $256 \times 256$ images. DWT has been used 
for decomposing. After decomposition approximation and detailed coefficients are produced. For improving contrast unsharp masking has been applied especially on approximation coefficients. For combining approximation coefficients that are produced after unsharp masking the average fusion rule is used. For merging detailed coefficients max fusion rule is used. IDWT has been used for reconstructing the final fused image.

\subsection{Discrete wavelet transform}

DWT has been one of the significant multi-scale and multi-resolution transform. Generally, spatial domain fusion produces spatial distortions and spectral degradations. The main advantage of DWT usage in image fusion is it reduces spectral degradations and spatial distortions produced in the fused image. Here in Fig. 1 DWT is used for decomposing two input source images (infrared, visible). Approximation coefficients and detailed coefficients are produced. Mainly approximation coefficients give average image information and detailed coefficients

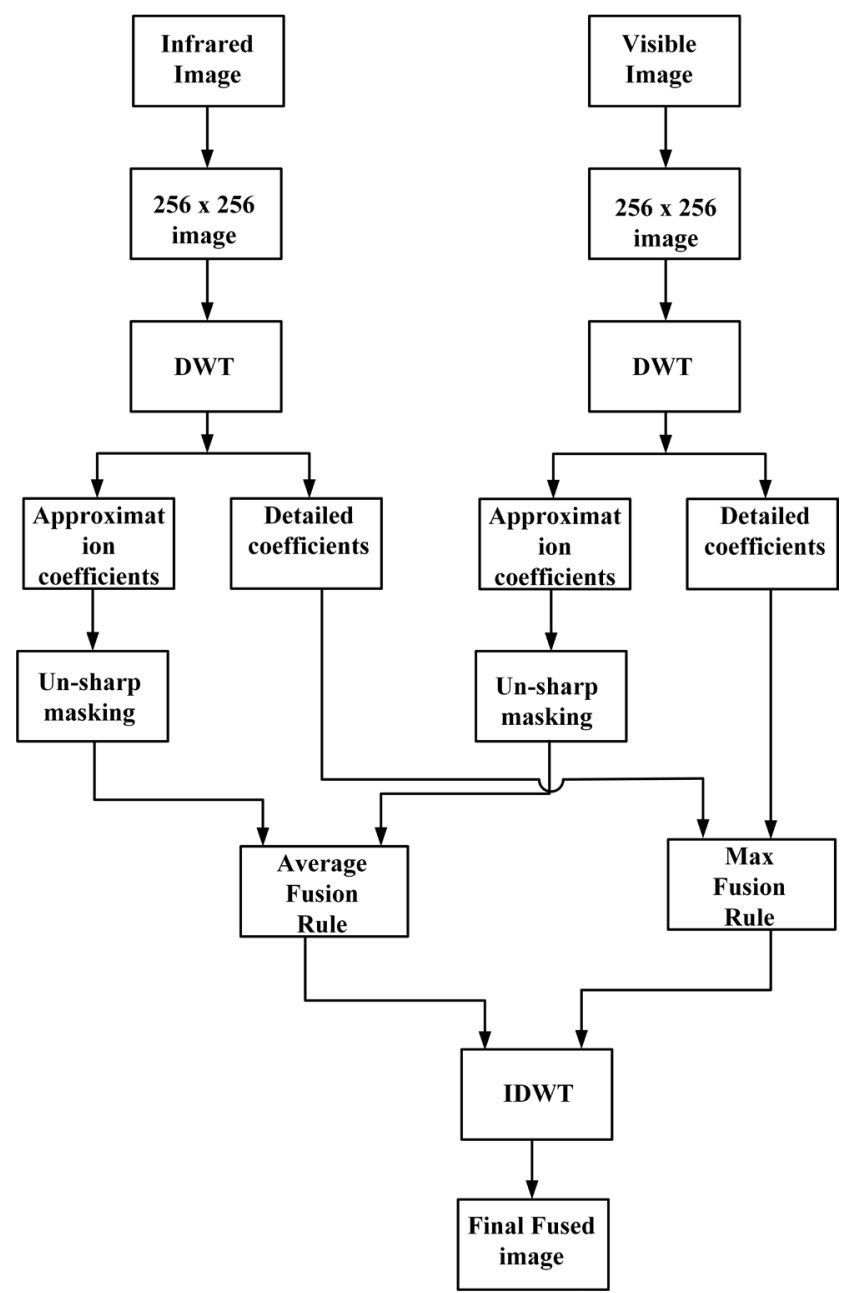

Fig. 1 Block diagram for the proposed method give edge details information. For merging approximation coefficients and detailed coefficients different fusion rules have been introduced. IDWT has been used for reconstructing the fused image.

\subsection{Unsharp masking}

Unsharp masking is an enhancement technique that will improve the appearance details of an image by increasing the contrast of edges. The blurred image is subtracted from its original image could produce a sharpened image.

Let $f(l, k)$ is a image, $f_{s}(l, k)$ is a sharpen image and $f^{\wedge}(l, k)$ is a blurred image of $f(l, k)$.

Therefore unsharp masking is obtained by

$f_{S}(l, k)=f(l, k)-f^{\wedge}(l, k)$.

As shown in Fig. 1 unsharp masking is applied to the approximation coefficients.

\subsection{Fusion rule that has been applied for approximation coefficients}

Unsharp masking is applied to approximation coefficients. Its main aim is to improve the contrast of edges and texture. Coefficients produced after un-sharp masking are fused by using average fusion rule. The main advantage of the average fusion rule is it considers all regions of both the images. The output of average fusion rule is calculated simply by adding corresponding coefficients of two images and dividing the result by two.

Let $I L f(v, w)$ is approximation fused coefficients, $I L_{1}(v, w)$ is approximation coefficients produced after unsharp masking for infrared image and $I L_{2}(v, w)$ is approximation coefficients produced after unsharp masking for the visible image. Average fusion rule is obtained by

$I L f(v, w)=\left[I L_{1}(v, w)+I L_{2}(v, w)\right] / 2$.

\subsection{Fusion rule that has been applied for detailed coefficients}

Generally, detailed coefficients contain edge information. For improving the focus of edge information max fusion rule has been introduced for merging detailed coefficients.

Let $\operatorname{IH} f(v, w)$ is detailed fused coefficients, $\operatorname{IH}_{1}(v, w)$ is detailed coefficients of infrared image, and $\mathrm{IH}_{2}(v, w)$ is detailed coefficients of the visible image. Max fusion rule is obtained by

$$
\operatorname{IHf}(v, w)=\max \left[I H_{1}(v, w), I H_{2}(v, w)\right] .
$$




\section{Experimental results and analysis}

\subsection{Performance metrics}

\subsubsection{Mean}

It is used for measuring average brightness information present in the fused image. Its value should be high for better performance. The formula for mean has been given by

$$
\mu=\frac{1}{(M * N)} \sum_{i=1}^{M} \sum_{j=1}^{N} G(i, j)
$$

where " $M$ " indicates the number of rows of fused image, " $N$ " indicates a number of columns of fused image, " $G(i, j)$ " indicates fused image, " $\mu$ " indicates mean.

\subsubsection{Entropy}

It is used for measuring information present in the fused image. Its value should be high for better performance. The formula for entropy has been given by

$$
E=-\sum_{j=0}^{L-1} h(j) \log h(j)
$$

where " $L$ " indicates a total number of grey levels, " $h(j)$ " indicates the probability density distribution of grey level $j$ and " $E$ " indicates entropy.

\subsubsection{Standard deviation}

It is used for measuring contrast. If the fused image is having high contrast then the standard deviation value is high. Its value should be high for better performance. The formula for standard deviation is given by

$$
\mathrm{SD}=\sqrt{\sum_{i=1}^{M} \sum_{j=1}^{N}(G(i, j)-\mu)^{2}}
$$

where " $M$ " indicates the number of rows of fused image, " $N$ " indicates a number of columns of fused image, "G(i,j)" indicates fused image, " $\mu$ " indicates mean and "SD" indicates standard deviation.

\subsubsection{Mean gradient}

The main aim of the mean gradient is to calculate the amount of edge information preserved in the fused image. If the fused image contains rich edge and texture information than the mean gradient value is high. Its value should be high for better performance. The formula for the mean gradient is given by

$$
\begin{aligned}
& \mathrm{MG}=\frac{1}{(M-1)(L-1)} \sum_{i=1}^{M-1} \sum_{j=1}^{N-1} \sqrt{\frac{A+B}{2}} \\
& A=[G(i, j)-G(i-1, j)]^{2}
\end{aligned}
$$

$B=[G(i, j)-G(i, j-1)]^{2}$

where " $M$ " indicates the number of rows of fused image, " $N$ " indicates a number of columns of fused image, "G(i,j)" indicates the fused image and "MG" indicates mean gradient.

\subsection{Subjective analysis of results}

Subjective analysis is done for evaluating performance. The proposed method results have been compared with existing techniques. The existing techniques that are used for comparison are

1. Implementation of DWT based fusion algorithm using sharpen filter [21].

2. Implementation of fusion algorithm in spatial domain using PCA, Histogram Equalization, Sharpen filter and Weighted average fusion rule [22].

3. Implementation of fusion algorithm in spatial domain using Histogram Equalization, Sharpen filter, PCA and Weighted average fusion rule [22].

The process for Implementation of DWT based fusion algorithm using sharpen filter is, a first infrared source image and visible source image are scaled into $256 \times 256$ images. Mainly infrared images are low contrast images. Sharpen filter is applied only on the infrared image, which produces an enhanced infrared image. The sharpen filter used here is laplacian filter. The main purpose of sharpen filter is to improve contrast. It highlights edge information. DWT is applied on enhanced infrared image and visible scaled input image. Approximation and detailed coefficients are produced. Weighted average rule has been used for merging both approximation coefficients and detailed coefficients. Weighted average rule is a simple and fast fusion rule, which will reduce noise in source images. IDWT is used for reconstructing the final fused-image. The $3 \times 3$ sharpen filter used in above method is shown in Fig. 2.

The process for Implementation of fusion algorithm in spatial domain using PCA, Histogram Equalization, Sharpen filter and Weighted average fusion rule is, a first

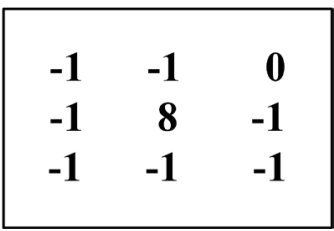

Fig. $23 \times 3$ sharpen filter 1 
infrared source image and visible source image are scaled into $256 \times 256$ images. Principle Component Analysis (PCA) has been applied to the scaled infrared image and visible image. PCA is mainly used for dimensional reduction. Histogram equalization is applied to the result of PCA of visible image. Histogram equalization is an enhancement technique that improves contrast of the image. Sharpen filter is applied to result of PCA of infrared image. Weighted average Fusion has been applied to results of histogram equalization and sharpen filter. The $3 \times 3$ sharpen filter used in above method is shown in Fig. 3.

The process for Implementation of fusion algorithm in spatial domain using Histogram Equalization, Sharpen

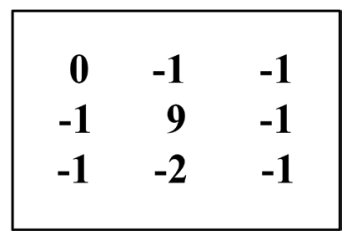

Fig. $33 \times 3$ sharpen filter 2 . filter, PCA and Weighted average fusion rule is, a first infrared source image and visible source image are scaled into $256 \times 256$ images. Histogram equalization is applied to the scaled visible image. Sharpen filter is applied on scaled infrared image. PCA is applied on result of Histogram equalization and sharpen filter of scaled visible and infrared input images. Weighted average fusion rule has been applied on result of PCA of both images. The $3 \times 3$ sharpen filter used in above method is shown in Fig. 3.

The dataset used for the experiment is TNO image fusion dataset. The experiment is conducted using MATLAB software. For doing the experiment the source images utilized are, first set of input infrared and visible images are of "Single person image" is shown in Fig. 4. The second set of input infrared and visible images are of "Two-person image" is shown in Fig. 5. And the third set of input infrared and visible images are of "Forest image" is shown in Fig. 6.

Fig. 4 shows the fused results of "Single person image", where Fig. 4 (a) shows an Infrared image, Fig. 4 (b) shows a visible image, Fig. 4 (c) shows sharpen fused-image which

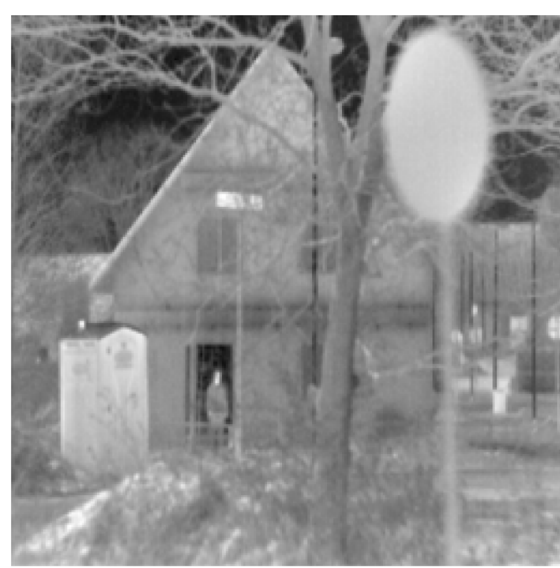

(a)

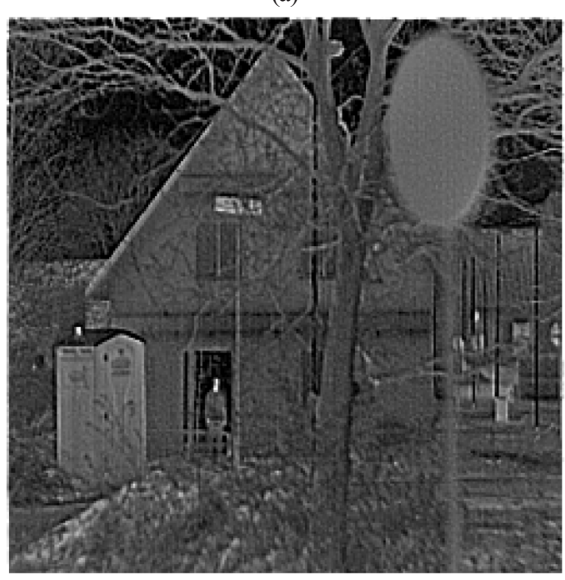

(d)

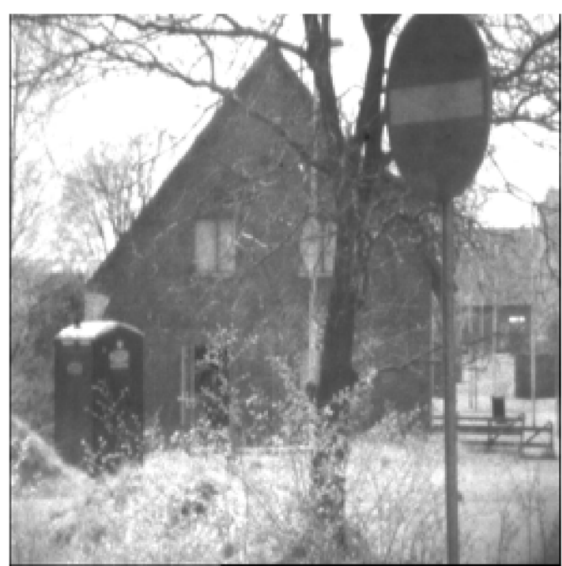

(b)

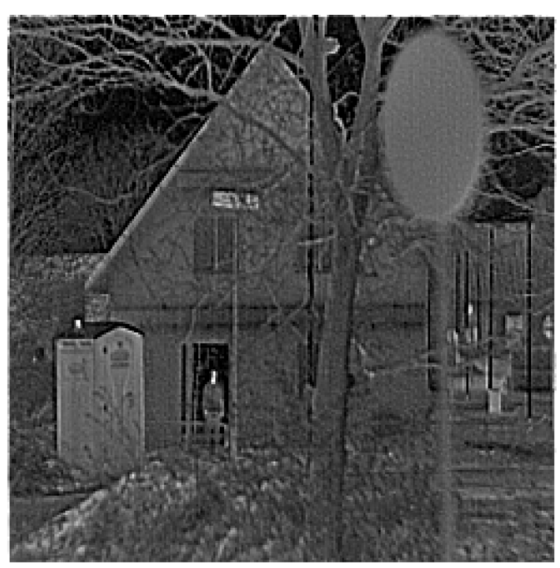

(e)

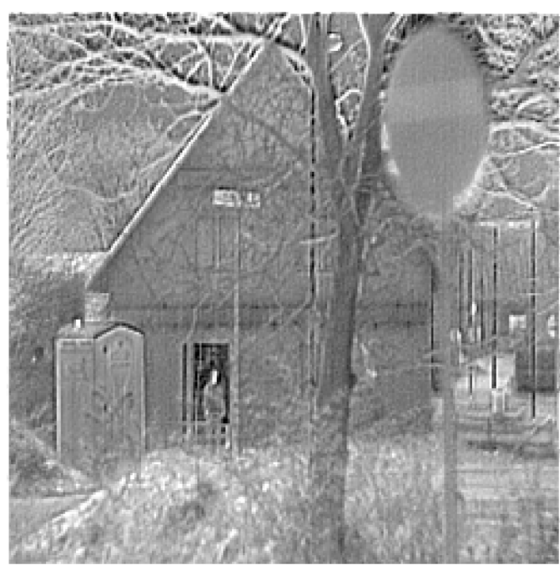

(c)

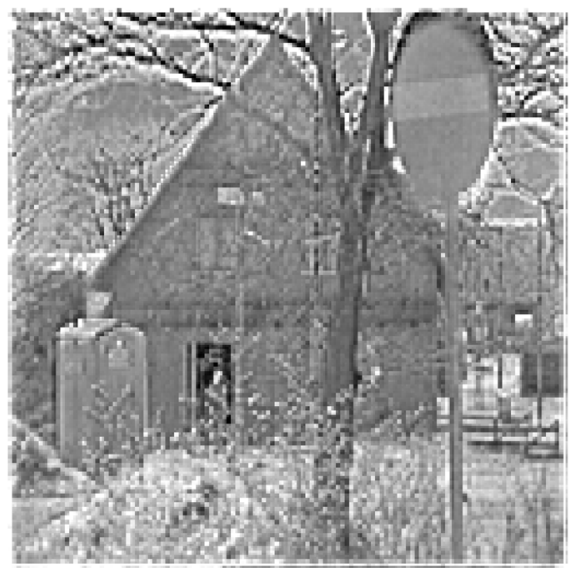

(f)

Fig. 4 The fused results of "Single person image"

(a) Infrared image (b) Visible image (c) Sharpen fused-image (d) PCA1fused-image (e) PCA2 fused-image (f) Proposed fused-image 


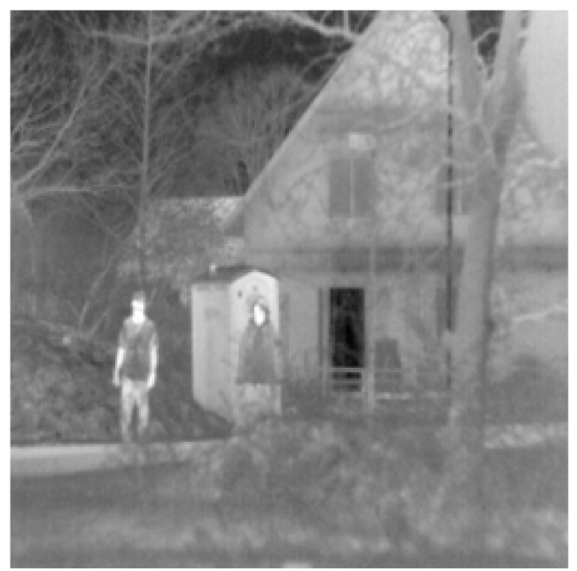

(a)

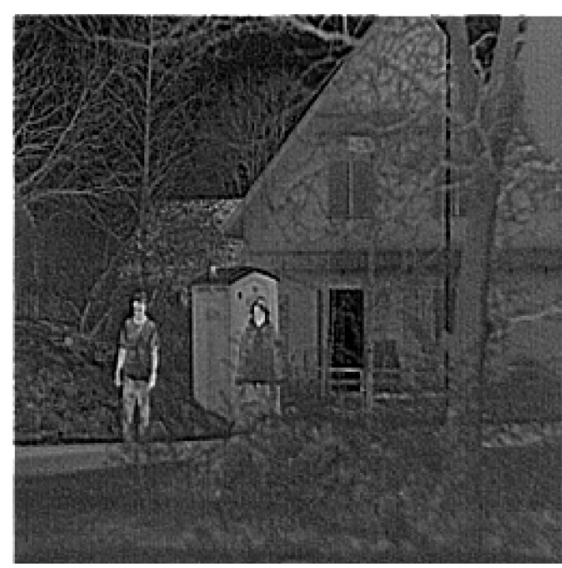

(d)

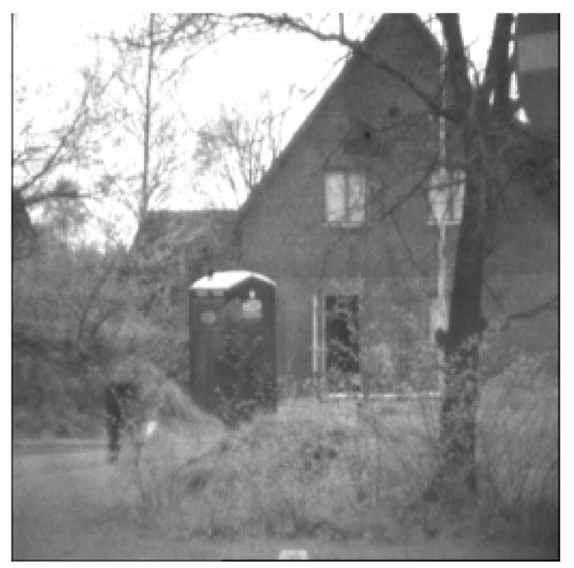

(b)

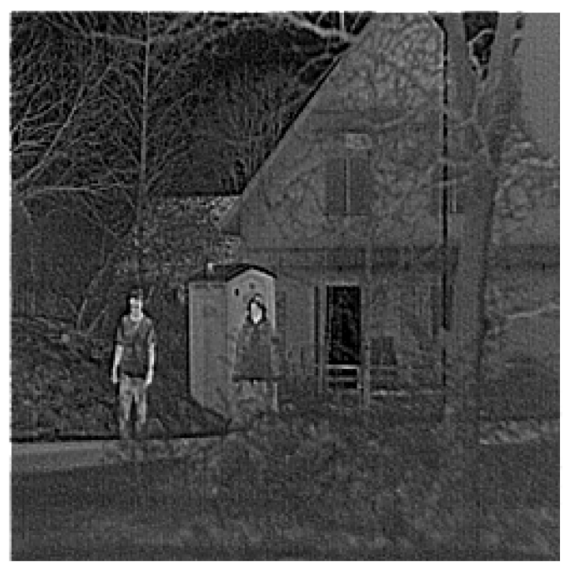

(e)

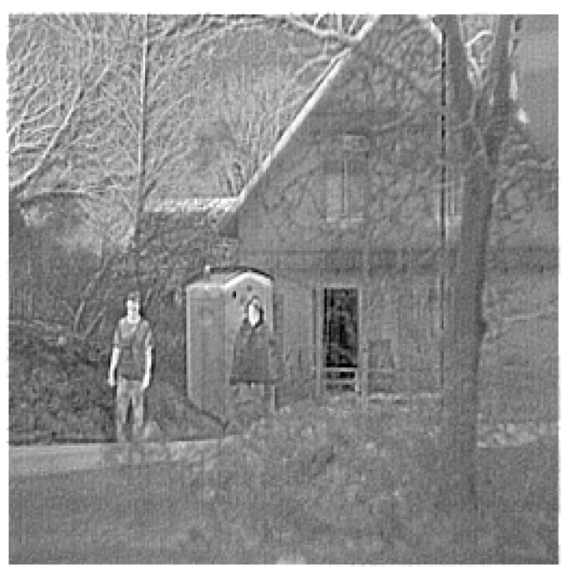

(c)

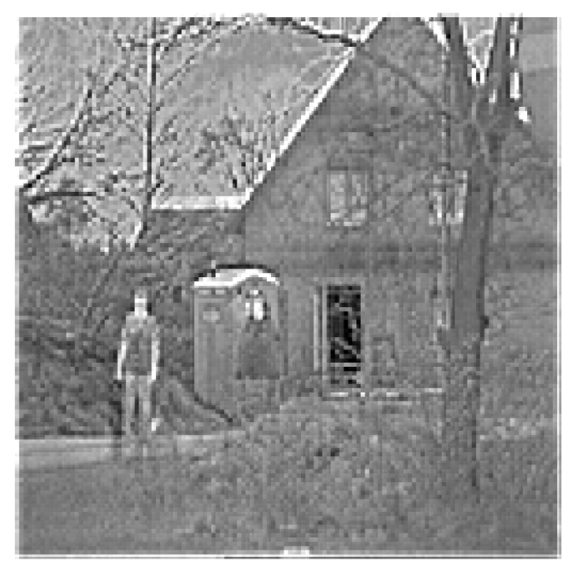

(f)

Fig. 5 The fused results of "Two-person image" (a) Infrared image (b) Visible image (c) Sharpen fused-image (d) PCA1fused-image (e) PCA2 fused-image (f) Proposed fused-image

is obtained after Implementation of DWT based fusion algorithm using sharpen filter, Fig. 4 (d) shows PCA1 fused-image which is obtained after Implementation of fusion algorithm in spatial domain using PCA, Histogram Equalization, Sharpen filter and Weighted average fusion rule, Fig. 4 (e) shows PCA2 fused-image which is obtained after Implementation of fusion algorithm in spatial domain using Histogram Equalization, Sharpen filter, PCA and Weighted average fusion rule and Fig. 4 (f) shows the proposed fused-image of "Single person image".

It can be observed that the proposed fused-image is having high contrast. Person, house, board and tree details are clearly seen in the proposed method as shown in Fig. 4 (f). But the above details are not properly seen in sharpen fused-image as shown in Fig. 4 (c) because in this method sharpen filter is only applied on infrared image, due to that visible source image features are less seen after fusion process. And also above details are not clearly seen in PCA1 fused-image and PCA2 fused-image as shown in Fig. 4 (d) and Fig. 4 (e) because weighted average fusion rule is applied in spatial domain due to that low brightness, low contrast and less quality fused images are produced.

Similarly Fig. 5 shows the fused results of "Two-person image", where Fig. 5 (a) shows an Infrared image, Fig. 5 (b) shows a visible image, Fig. 5 (c) shows sharpen fused-image which is obtained after Implementation of DWT based fusion algorithm using sharpen filter, Fig. 5 (d) shows PCA1 fused-image which is obtained after Implementation of fusion algorithm in spatial domain using PCA, Histogram Equalization, Sharpen filter and Weighted average fusion rule, Fig. 5 (e) shows PCA2 fused-image which is obtained after Implementation of fusion algorithm in spatial domain using Histogram Equalization, Sharpen filter, PCA and Weighted average fusion rule and Fig. 5 (f) shows the proposed fused-image of "Two-person image".

It can be observed that two persons, house, board and tree details are not clearly seen in the sharpen fused-image, PCA1 fused-image, and PCA2 fused-image as shown in Fig. 5 (c)-(e) due to poor texture information. 


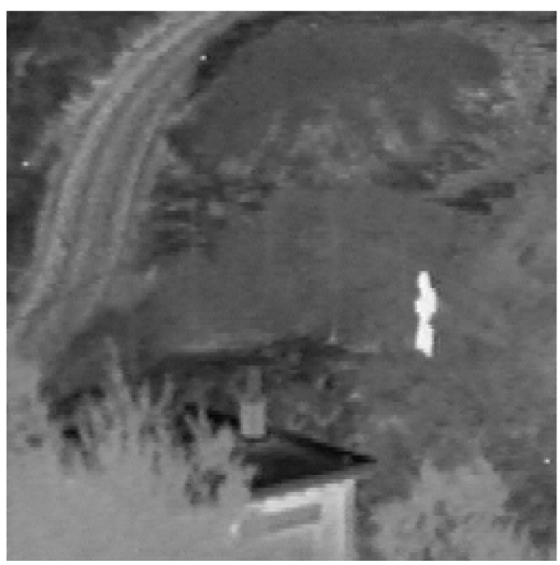

(a)

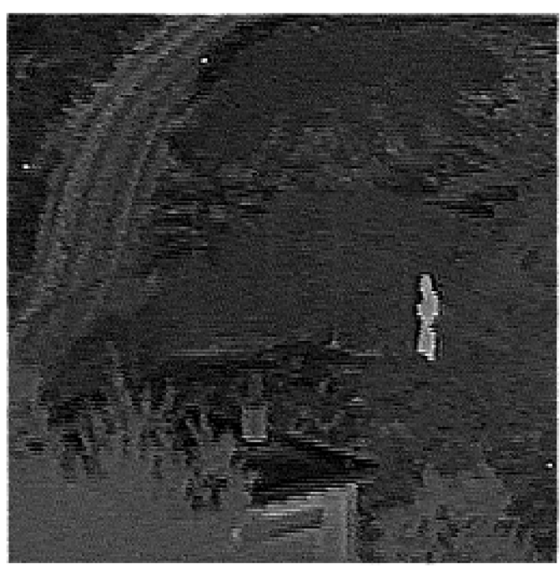

(d)

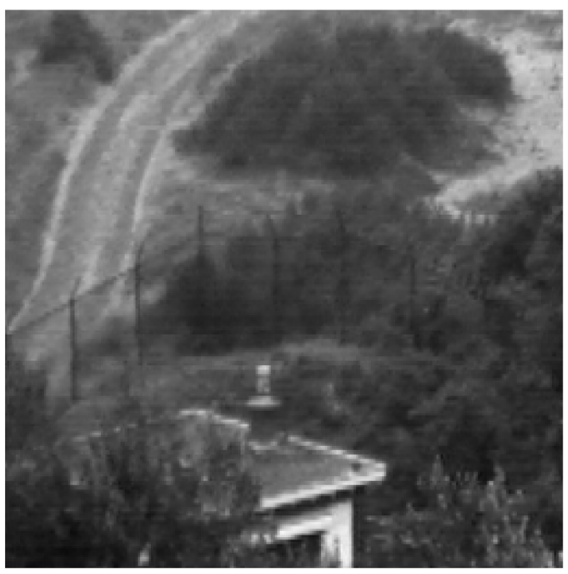

(b)

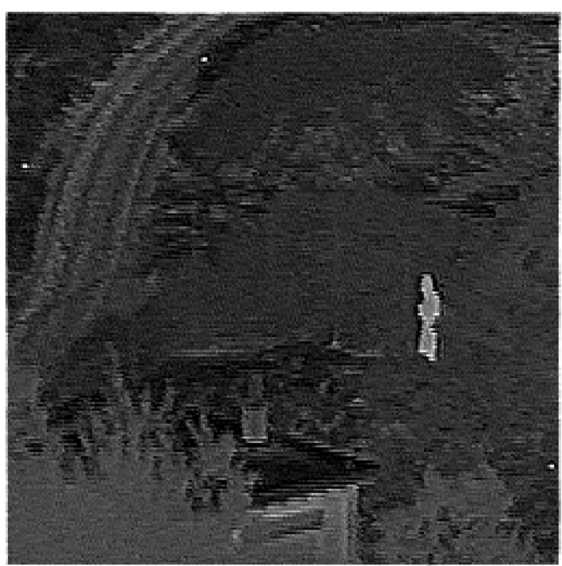

(e)

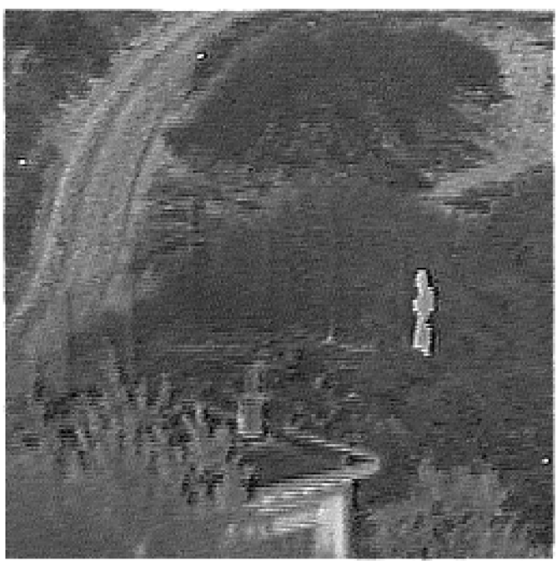

(c)

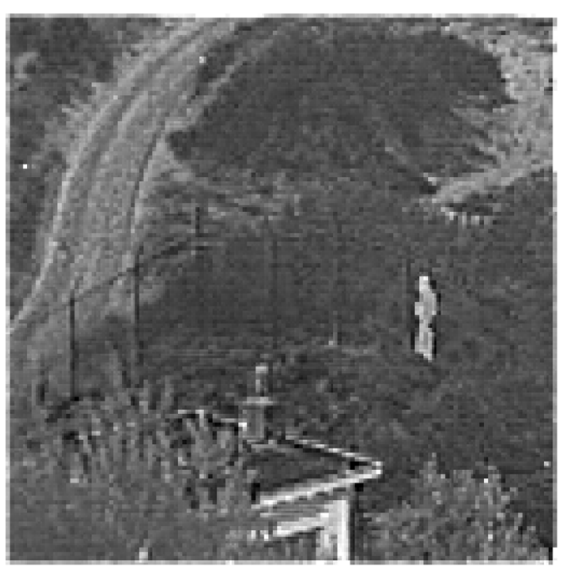

(f)

Fig. 6 The fused results of "Forest image"

(a) Infrared image (b) Visible image (c) Sharpen fused-image (d) PCA1fused-image (e) PCA2 fused-image (f) Proposed fused-image

Fused-image obtained using the proposed method is providing good contrast, texture, and edge information because of that person, house, board and tree details are clearly seen in Fig. 5 (f).

Similarly Fig. 6 shows the fused results of "Forest image", where Fig. 6 (a) shows an Infrared image, Fig. 6 (b) shows a visible image, Fig. 6 (c) shows sharpen fused-image which is obtained after Implementation of DWT based fusion algorithm using sharpen filter, Fig. 6 (d) shows PCA1 fused-image which is obtained after Implementation of fusion algorithm in spatial domain using PCA, Histogram Equalization, Sharpen filter and Weighted average fusion rule, Fig. 6 (e) shows PCA2 fused-image which is obtained after Implementation of fusion algorithm in spatial domain using Histogram Equalization, Sharpen filter, PCA and Weighted average fusion rule and Fig. 6 (f) shows the proposed fused-image of "Forest image".

Similarly, it can be observed that person, plants and fence details are not clearly seen in the sharpen fused-image, PCA1 fused-image, and PCA2 fused-image as shown in Fig. 6 (c)-(e) due to poor texture and edge information. Whereas person, plants and fence details are clearly seen in the proposed fused image as shown in Fig. 6 (f). By comparing all methods we can observe that the proposed fused image is providing better contrast, scene, and target information.

\subsection{Objective analysis of results}

Objective Analysis has been done especially for evaluating performance. The metrics used for measuring performance are mean, entropy, standard deviation and mean gradient.

Tables 1, 2 and 3 show the comparison results for "Single person image", "Two-person image" and "Forest image". From Tables 1, 2 and 3 it can be seen that the proposed method got better values than existing techniques with reference to mean, entropy and standard deviation. Here better values are highlighted with bold. 
Table 1 Comparison results for "Single person image"

\begin{tabular}{lcccc}
\hline Method & Mean & Entropy & $\begin{array}{c}\text { Standard } \\
\text { Deviation }\end{array}$ & $\begin{array}{c}\text { Mean } \\
\text { gradient }\end{array}$ \\
\hline Sharpen & 147.1593 & 7.1003 & 39.9328 & 18.7731 \\
PCA1 & 71.6086 & 6.8065 & 43.0949 & 19.8960 \\
PCA2 & 71.1319 & 6.8023 & 43.0949 & 19.8960 \\
Proposed & $\mathbf{1 4 8 . 3 9 1 9}$ & $\mathbf{7 . 2 7 5 7}$ & $\mathbf{4 7 . 2 5 2 1}$ & $\mathbf{2 0 . 7 3 9 9}$ \\
\hline
\end{tabular}

Table 2 Comparison results for "Two-person image"

\begin{tabular}{lcccc}
\hline Method & Mean & Entropy & $\begin{array}{c}\text { Standard } \\
\text { Deviation }\end{array}$ & $\begin{array}{c}\text { Mean } \\
\text { gradient }\end{array}$ \\
\hline Sharpen & 134.9493 & 6.7858 & 33.4370 & 15.4057 \\
PCA1 & 66.7068 & 6.6406 & 22.9660 & $\mathbf{1 6 . 5 6 4 7}$ \\
PCA2 & 66.2168 & 6.6386 & 22.9660 & $\mathbf{1 6 . 5 6 4 7}$ \\
Proposed & $\mathbf{1 3 5 . 7 7 0 2}$ & $\mathbf{6 . 9 7 7 1}$ & $\mathbf{3 9 . 9 7 7 4}$ & 15.6680 \\
\hline
\end{tabular}

Table 3 Comparison results for "Forest image"

\begin{tabular}{lcccc}
\hline Method & Mean & Entropy & $\begin{array}{c}\text { Standard } \\
\text { Deviation }\end{array}$ & $\begin{array}{c}\text { Mean } \\
\text { gradient }\end{array}$ \\
\hline Sharpen & 92.7952 & 6.7825 & 33.6649 & 17.5139 \\
PCA1 & 51.4803 & 6.5179 & 32.8658 & $\mathbf{1 9 . 7 0 6 1}$ \\
PCA2 & 71.1319 & 6.2605 & 32.8658 & $\mathbf{1 9 . 7 0 6 1}$ \\
Proposed & $\mathbf{9 3 . 9 0 7 4}$ & $\mathbf{6 . 7 9 9 1}$ & $\mathbf{3 4 . 5 6 2 9}$ & 10.8214 \\
\hline
\end{tabular}

Average values of proposed method and existing techniques with reference to mean, entropy, standard deviation and mean gradient are shown in Table 4. Proposed method got better results with reference to mean, entropy and standard deviation.

Figs. 7-10 shows plots of different fusion methods compared with reference to mean, entropy, standard deviation and mean gradient.

From Figs. 7-9 we can observe better results are achieved by the proposed method than the existing techniques.

\section{Conclusion}

Discrete wavelet transform based image fusion using unsharp masking has been proposed. First input images are scaled to $256 \times 256$ images. DWT decomposition has been used. Approximation and detailed coefficients have been produced. Here for improving contrast unsharp masking has been applied on approximation coefficients.

Table 4 Comparison of average results of different fusion methods

\begin{tabular}{lcccc}
\hline Method & Mean & Entropy & $\begin{array}{c}\text { Standard } \\
\text { Deviation }\end{array}$ & $\begin{array}{c}\text { Mean } \\
\text { gradient }\end{array}$ \\
\hline Sharpen & 124.9679 & 6.8895 & 35.6782 & 17.2309 \\
PCA1 & 63.2652 & 6.6550 & 32.9755 & $\mathbf{1 8 . 7 2 2 2}$ \\
PCA2 & 69.4935 & 6.5671 & 32.9755 & $\mathbf{1 8 . 7 2 2 2}$ \\
Proposed & $\mathbf{1 2 6 . 0 2 3 1}$ & $\mathbf{7 . 0 1 7 3}$ & $\mathbf{4 0 . 5 9 7 4}$ & 15.7431 \\
\hline
\end{tabular}

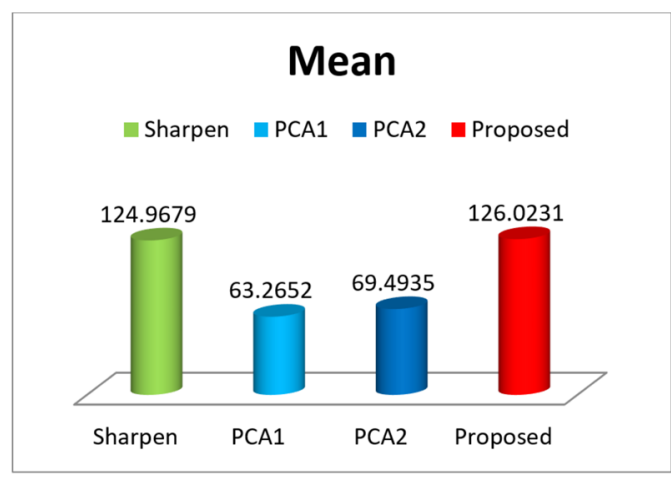

Fig. 7 Different fusion methods comparison with reference to Mean

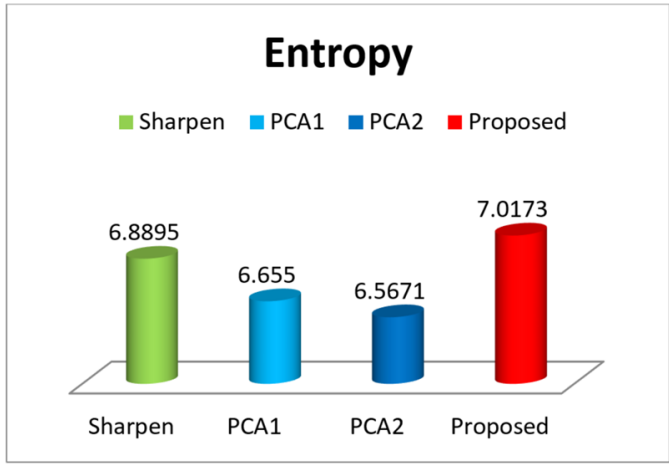

Fig. 8 Different fusion methods comparison with reference to Entropy

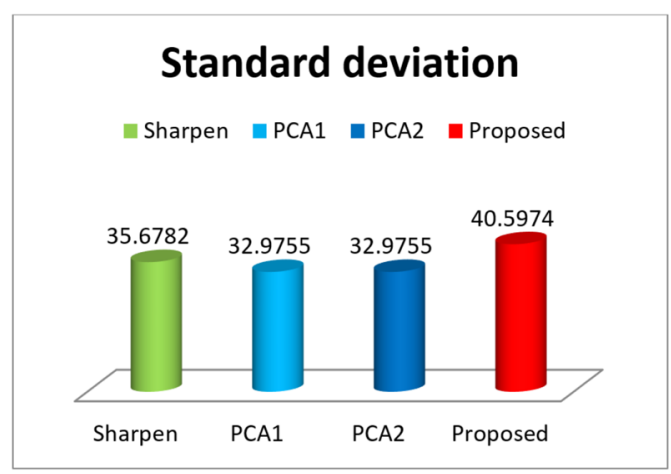

Fig. 9 Different fusion methods comparison with reference to Standard deviation

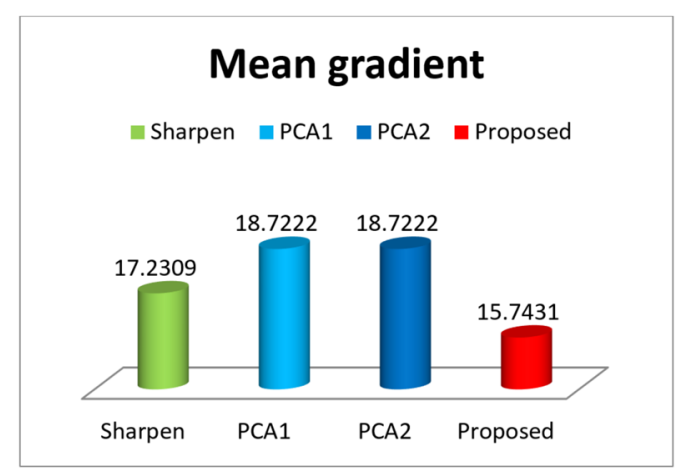

Fig. 10 Different fusion methods comparison with reference to Mean gradient 
For combining approximation (low-frequency) coefficients produced after unsharp masking fusion rule especially used is average fusion rule. Max fusion rule has been introduced for merging detailed coefficients. IDWT is used for reconstructing the fused image.

Comparison of results have been done between the proposed method and existing techniques. The existing techniques that are used for comparison are

1. Implementation of DWT based fusion algorithm using sharpen filter [21].

2. Implementation of fusion algorithm in spatial domain using PCA, Histogram Equalization, Sharpen filter and Weighted average fusion rule [22].

\section{References}

[1] Xu, F., Zeng, D., Zhang, J., Zheng, Z., Wei, F., Wang, T. "Detail enhancement of blurred infrared images based on frequency extrapolation", Infrared Physics \& Technology, 76, pp. 560-568, 2016. https://doi.org/10.1016/j.infrared.2016.04.008

[2] Liu, Z., Feng, Y., Zhang, Y., Li, X. "A fusion algorithm for infrared and visible images based on RDU-PCNN and ICA-bases in NSST domain", Infrared Physics \& Technology, 79, pp. 183-190, 2016. https://doi.org/10.1016/j.infrared.2016.10.015

[3] Ma, J., Ma, Y., Li, C. "Infrared and visible image fusion methods and applications: A survey", Information Fusion, 45, pp. 153-178, 2019. https://doi.org/10.1016/j.inffus.2018.02.004

[4] Muller, A. C., Narayanan, S. "Cognitively-engineered multisensor image fusion for military applications", Information Fusion, 10(2), pp. 137-149, 2009.

https://doi.org/10.1016/j.inffus.2008.08.008

[5] Feng, Z. J., Zhang, X. L., Yuan, L. Y., Wang, J. N. "Infrared Target Detection and Location for Visual Surveillance Using Fusion Scheme of Visible and Infrared Images", Mathematical Problems in Engineering, 2013, Article ID: 720979, 2013.

https://doi.org/10.1155/2013/720979

[6] Chang, X., Jiao, L., Liu, F., Xin, F. "Multicontourlet-Based Adaptive Fusion of Infrared and Visible Remote Sensing Images", IEEE Geoscience and Remote Sensing Letters, 7(3), pp. 549-553, 2010. https://doi.org/10.1109/LGRS.2010.2041323

[7] Hanna, B. V., Gorbach, A. M., Gage, F. A., Pinto, P. A., Silva, J. S., Gilfillan, L. G., Kirk, A. D., Elster, E. A. "Intraoperative Assessment of Critical Biliary Structures with Visible Range/ Infrared Image Fusion", Journal of the American College of Surgeons, 206(6), pp. 1227-1231, 2008.

https://doi.org/10.1016/j.jamcollsurg.2007.10.012

[8] May, K. A., Georgeson, M. A. "Blurred edges look faint, and faint edges look sharp: The effect of a gradient threshold in a multi-scale edge coding model", Vision Research, 47(13), pp. 1705-1720, 2007. https://doi.org/10.1016/j.visres.2007.02.012

[9] Li, H., Liu, L., Huang, W., Yue, C. "An improved fusion algorithm for infrared and visible images based on multi-scale transform", Infrared Physics and Technology, 74, pp. 28-37, 2016.

https://doi.org/10.1016/j.infrared.2015.11.002
3. Implementation of fusion algorithm in spatial domain using Histogram Equalization, Sharpen filter, PCA and Weighted average fusion rule [22].

The proposed method fused image is having higher contrast when compared with fused images obtained using existing techniques. And it is also giving better results with reference to mean, entropy and standard deviation when compared to existing techniques. The proposed method is providing good texture and edge information so that scene information can easily be understood by humans.

[10] Zhan, L., Zhuang, Y., Huang, L. "Infrared and Visible Images Fusion Method Based On Discrete Wavelet Transform", Journal of Computers, 28(2), pp. 57-71, 2017. https://doi.org/10.3966/199115592017042802005

[11] Zhou, Z., Lin, J., Jin, W., Peng, Z., Pinglv, Y. "Image fusion by combining SWT and variational model", In: 2011 4th International Congress on Image and Signal Processing, Shanghai, China, 2011, pp. 1907-1910. https://doi.org/10.1109/CISP.2011.6100633

[12] Paramanandham, N., Rajendiran, K. "Infrared and visible image fusion using discrete cosine transform and swarm intelligence for surveillance applications", Infrared Physics \& Technology, 88, pp. 13-22, 2018. https://doi.org/10.1016/j.infrared.2017.11.006

[13] Liu, Z., Feng, Y., Chen, H., Jaio, L. "A fusion algorithm for infrared and visible based on guided filtering and phase congruency in NSST domain", Optics and Lasers in Engineering, 97, pp. 71-77, 2017. https://doi.org/10.1016/j.optlaseng.2017.05.007

[14] Li, H., Manjunath, B. S., Mitra, S. K. "Multisensor Image Fusion Using the Wavelet Transform", Graphical Models and Image Processing, 57(3), pp. 235-245, 1995. https://doi.org/10.1006/gmip.1995.1022

[15] Fan, L., Zhang, Y., Zhou, Z., Semanek, D. P., Wang, S., Wu, L. "An Improved Image Fusion Algorithm Based on Wavelet Decomposition", Journal of Convergence Information Technology, 5(10), pp. 15-21, 2010. [online] Available at: http://citeseerx.ist.psu. edu/viewdoc/download; jsessionid=511E898EA66A614FE4487D$510483325 \mathrm{~A}$ ?doi=10.1.1.501.9698\& rep=rep1\&type=pdf [Accessed: 08 June 2019]

[16] Godse, D. A., Bormane, D. S. "Wavelet based image fusion using pixel based maximum selection rule", International Journal of Engineering Science and Technology, 3(7), pp. 5572-5577, 2011. [online] Available at: https://www.researchgate.net/profile/D_Bormane/publication/266016648_WAVELET_BASED_ IMAGE_FUSION_USING_PIXEL_BASED_MAXIMUM_ SELECTION_RULE/links/552d23360 cf21acb092137bb/ WAVELET-BASED-IMAGE-FUSION-USING-PIXEL-BASEDMAXIMUM-SELECTION-RULE.pdf [Accessed: 11 June 2019] 
[17] Xu, F., Su, S. "An Enhanced Infrared and Visible Image Fusion Method Based on Wavelet Transform", In: 2013 5th International Conference on Intelligent Human-Machine Systems and Cybernetics, Hangzhou, China, 2013, pp. 453-456. https://doi.org/10.1109/IHMSC.2013.255

[18] Zhou, Z. H., Tan, M. "Infrared Image and Visible Image Fusion Based on Wavelet Transform", Advanced Materials Research, 756759, pp. 2850-2856, 2013.

https://doi.org/10.4028/www.scientific.net/AMR.756-759.2850

[19] Vijayarajan, R., Muttan, S. "Discrete wavelet transform based principal component averaging fusion for medical images", AEU-International Journal of Electronics and Communications, 69(6), pp. 896-902, 2015. https://doi.org/10.1016/j.aeue.2015.02.007

[20] Han, X., Zhang, L. L., Du, L. Y., Huan, K. W., Shi, X. G. "Fusion of infrared and visible images based on discrete wavelet transform", In: Selected Papers of the Photoelectronic Technology Committee Conferences, Hefei, Suzhou, and Harbin, China, Vol. 9795, 2015, Article number: 979510.

https://doi.org/10.1117/12.2216054
[21] Habeeb, N. J., Omran, S. H., Radih, D. A. "Contrast Enhancement for Visible-Infrared Image Using Image Fusion and Sharpen Filters", In: 2018 International Conference on Advanced Science and Engineering (ICOASE), Duhok, Iraq, 2018, pp. 64-69. https://doi.org/10.1109/ICOASE.2018.8548898

[22] Habeeb, N. J., Al-Taei, A., Fadhil-Ibrahim, M. "Contrast Enhancement for Multi-Modality Image Fusion in Spatial Domain", Journal of Theoretical and Applied Information Technology, 96(20), pp. 6926-6936, 2018. [online] Available at: https://www.semanticscholar.org/paper/CONTRASTENHANCEMENT-FOR-MULTIMODALIT Y-IMAGEFUSION-Habeeb-A1-Taei/355a748c9d7f5adf8f87c9e93092adaf5 b609b0b [Accessed: 13 September 2019] 